\title{
DETERMINAÇÃO TURBIDIMÉTRICA DE DIPIRONA EM FLUXO UTILIZANDO UM REATOR CONTENDO CLORETO DE PRATA IMOBILIZADO EM RESINA POLIÉSTER
}

Luiz H. Marcolino-Jr., Viviane Gomes Bonifácio e Orlando Fatibello-Filho*

Departamento de Química, Centro de Ciências Exatas e de Tecnologia, Universidade Federal de São Carlos, CP 676, 13560-970 São Carlos - SP

Marcos F. S. Teixeira

Universidade Estadual Paulista, Unidade São Vicente, 11330-900 São Vicente - SP

Recebido em 6/5/04; aceito em 11/2/05; publicado na web em 30/6/05

\begin{abstract}
FLOW INJECTION TURBIDIMETRIC DETERMINATION OF DIPYRONE USING A SOLID-PHASE REACTOR CONTAINING SILVER CHLORIDE IMMOBILIZED IN A POLYESTER RESIN. A simple flow injection procedure was developed for determining dipyrone (1-phenyl-2,3-dimethyl-4-methylaminomethano-5-pyrazolone sodium, metamizol, analgin) in pharmaceutical formulations. The determination is based on the reduction of $\mathrm{Ag}^{+}$ions to $\mathrm{Ag}^{0}$ by dipyrone. A colloidal suspension of $\mathrm{Ag}^{0} \mathrm{produced}$ was transported by carrier solution $\left(0.01 \mathrm{~mol} \mathrm{~L}^{-1} \mathrm{NaOH}\right)$ and turbidimetrically detected at $425 \mathrm{~nm}$. The analytical curve for dipyrone was linear in the range from $5.0 \times 10^{-4}$ to $2.5 \times 10^{-3} \mathrm{~mol} \mathrm{~L}^{-1}$ with a correlation coefficient of 0.9990 . The detection limit was $1.3 \times 10^{-4} \mathrm{~mol} \mathrm{~L}^{-1}$ $\left(3 \sigma_{\mathrm{B}} /\right.$ slope). The relative standard deviation for ten successive measurements was $1.8 \%$ and an analytical frequency of $45 \mathrm{~h}^{-1}$ was obtained. The recovery values from three samples ranged from 96.0 to $102 \%$.
\end{abstract}

Keywords: dipyrone; flow injection turbidimetry; solid-phase reactor.

\section{INTRODUÇÃO}

A dipirona (sal de sódio do 1-fenil-2,3-dimetil-4-metilaminometanossulfonato-5-pirazolona) é um pó cristalino branco, solúvel em água e álcool, que apresenta atividades analgésicas e antipiréticas ${ }^{1}$. Essa droga é rapidamente absorvida após administração oral e amplamente metabolizada. Quimicamente, a dipirona é um derivado 5-pirazolônico com a presença de um grupo metanossulfônico na estrutura (Figura 1). É comercializada principalmente na forma sódica (solução oral, injetável e comprimidos) sendo que $80 \%$ das vendas são feitas sem prescrição médica. Existem no país cerca de 125 produtos à base de dipirona, sendo $71 \mathrm{em}$ associação a outras substâncias.

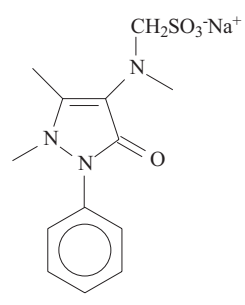

Figura 1. Estrutura molecular da dipirona

Diversos procedimentos são descritos na literatura para determinação de dipirona. Vassileva-Alexandrova e Shishmanov² propuseram um procedimento para determinação de dipirona baseado na reação desse fármaco com o reagente cloreto de neotetrazólio, produzindo um composto amarelo solúvel em meio de etanol-HCl. O método mostrou-se bastante seletivo e preciso na determinação de dipirona em produtos farmacêuticos, em amostras contendo de 1,0 a $5,0 \mu \mathrm{g} \mathrm{mL} \mathrm{m}^{-1}$ do fármaco.

*e-mail: bello@dq.ufscar.br
Sakiara et al. $^{3}$ desenvolveram um método baseado na oxidação seletiva da dipirona na presença de ácido sulfúrico, promovendo a formação de formaldeído, que reage com o ácido cromotrópico produzindo um composto violeta, monitorado espectrofotometricamente em $575 \mathrm{~nm}$. O procedimento apresentou uma resposta linear no intervalo de concentração de dipirona de 0,57 a $5,7 \mathrm{mg} \mathrm{L}^{-1}$ $\left(1,6 \times 10^{-6}\right.$ a $\left.1,6 \times 10^{-5} \mathrm{~mol} \mathrm{~L}^{-1}\right)$ mostrando-se preciso e seletivo na determinação de dipirona em produtos farmacêuticos.

Bautista et al..$^{4}$ determinaram a dipirona indiretamente, utilizando um reator em fase sólida contendo $\mathrm{PbO}_{2}$ acoplado a um sistema de análise por injeção em fluxo. O método é baseado na reação do fármaco no reator, promovendo simultaneamente a oxidação da dipirona e a liberação de íons $\mathrm{Pb}(\mathrm{II})$. Estes íons agem como catalizadores na reação entre vermelho de pirogalol e perssulfato de potássio, resultando na diminuição da coloração da solução. A diminuição da absorbância foi monitorada em 520 $\mathrm{nm}$ e a freqüência analítica obtida foi de 72 determinações por hora. Com esse procedimento, foi possível determinar dipirona no intervalo de concentração de $5,7 \times 10^{-6}$ a $4,6 \times 10^{-5} \mathrm{~mol} \mathrm{~L}^{-1}$.

A determinação espectrofotométrica proposta por Qureshi et al. ${ }^{5}$ baseia-se na redução de íons Fe(III) por ação da dipirona e subseqüente complexação dos íons Fe(II) com 1,10fenantrolina, que produz um complexo vermelho, monitorado em $500 \mathrm{~nm}$. Esse mesmo grupo de pesquisadores desenvolveu outro procedimento onde a dipirona foi oxidada por uma solução de iodato de potássio em meio ácido, produzindo um composto de coloração amarela, monitorado espectrofotometricamente em $460 \mathrm{~nm}^{6}$.

Vários outros métodos são descritos na literatura para determinação de dipirona por detecção espectrofotométrica ${ }^{7-11}$, fluorimétrica $^{12}$, eletroquímica ${ }^{13-15}$, por quimiluminescência ${ }^{16-18} \mathrm{e}$ HPLC $^{19-23}$. Entretanto, alguns desses procedimentos são de alto custo, enquanto outros são trabalhosos e/ou requerem profissionais com boa capacitação, pois muitos destes procedimentos envolvem diversas etapas analíticas. 
O emprego de reagentes em fase sólida (RFS) acoplados a sistemas de análise por injeção em fluxo tem sido amplamente explorado atualmente. Os RFS têm sido usados para diferentes propósitos, incluindo pré-concentração, conversão de amostras, imobilização de enzimas e produção in situ de reagentes instáveis ${ }^{24}$.

Neste trabalho, um reator em fase sólida contendo $\mathrm{AgCl}$ foi inserido em um sistema de análise por injeção em fluxo de linha única, para atuar como fonte de íons $\mathrm{Ag}^{+}$, e aplicado na determinação de dipirona. $\mathrm{O}$ procedimento está baseado na propriedade redutora da dipirona. Os íons $\mathrm{Ag}^{+}$contidos no reator são reduzidos pela dipirona a $\mathrm{Ag}^{0}$ que é, então, detectada turbidimetricamente em $425 \mathrm{~nm}$. A concentração de dipirona injetada no sistema é proporcional à quantidade de $\mathrm{Ag}^{0}$ gerada.

\section{PARTE EXPERIMENTAL}

\section{Equipamentos}

Um diagrama esquemático do sistema em fluxo é mostrado na Figura 2. Para propulsão das soluções de referência, amostras e solução transportadora foi utilizada uma bomba peristáltica Ismatec de 12 canais, modelo 7618-50 com tubos de propulsão de Tygon ${ }^{\circledR}$ de diferentes diâmetros internos; tubos de polietileno de $0,8 \mathrm{~mm}$ de diâmetro interno e conectores de acrílico. As soluções de referência ou amostras foram injetadas utilizando um injetor-comutador manual duplo construído em acrílico ${ }^{25}$. Todas as medidas espectrofotométricas foram obtidas utilizando-se um espectrofotômetro de feixe simples Femto, modelo 435 equipado com uma célula espectrofotométrica com caminho ótico de $1,00 \mathrm{~cm}$, acoplado a um registrador Cole Parmer modelo 1202-000.

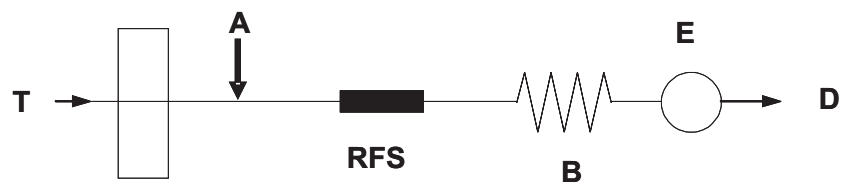

Figura 2. Diagrama esquemático do sistema de análise por injeção em fluxo para determinação turbidimétrica de dipirona. $\boldsymbol{T}$, solução transportadora

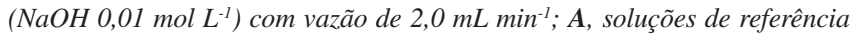
ou amostra (alça de amostragem $=375 \mu L) ; \boldsymbol{R F S}$, reator em fase sólida contendo $\mathrm{AgCl}(8,5 \mathrm{~cm} \times 2,0 \mathrm{~mm}$ d.i.); $\boldsymbol{B}$, bobina reacional $(50 \mathrm{~cm}) ; \boldsymbol{E}$, espectrofotômetro (425 $\mathrm{nm}) ; \boldsymbol{D}$, descarte

\section{Reagentes e soluções}

Dipirona sódica (Boheringer Ingelheim), $\mathrm{NaOH}$ (Merck), resina poliéster (Resapol T-208, Resana) e peróxido de metiletilcetona (Iberê, Ramires \& Cia) foram utilizados neste trabalho.

Foram preparadas várias soluções de $\mathrm{NaOH}$ em diferentes concentrações, dissolvendo-se massas adequadas dessa base em água desionizada. A solução estoque de dipirona $10 \mathrm{mmol} \mathrm{L}^{-1}$ foi preparada diariamente dissolvendo-se $0,1757 \mathrm{~g}$ de dipirona em $50 \mathrm{~mL}$ de água desionizada. Soluções contendo entre $5,0 \times 10^{-4}$ e $2,5 \times 10^{-3} \mathrm{~mol}$ $\mathrm{L}^{-1}$ de dipirona foram preparadas por diluição da solução estoque em água desionizada.

\section{Determinação de dipirona em amostras comerciais}

Amostras contendo dipirona foram adquiridas no comércio e analisadas pelo método proposto.

Alíquotas de $700 \mu \mathrm{L}$ das soluções foram transferidas para balões volumétricos de $100 \mathrm{~mL}$ e diluídas para o volume com água desionizada. Diluições posteriores foram realizadas até a obtenção de uma concentração final teórica de $1,0 \times 10^{-3} \mathrm{~mol} \mathrm{~L}^{-1}$ de dipirona.

\section{Método comparativo}

Os resultados obtidos pelo método proposto foram comparados com aqueles obtidos pelo método oficial da farmacopéia brasileira ${ }^{26}$ que consiste na titulação iodimétrica de dipirona, baseada na oxidação do grupo metanossulfônico a sulfato em meio ácido, de acordo com a reação:

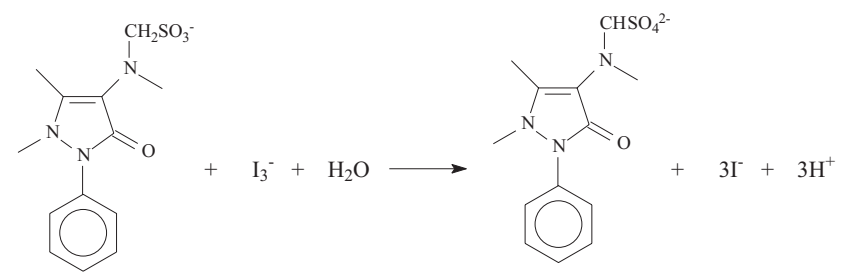

Cada 1,0 mL da solução de iodo $0,05 \mathrm{~mol} \mathrm{~L}^{-1}$ corresponde a 16,67 mg de dipirona sódica.

\section{Preparação e imobilização do $\mathrm{AgCl}_{(\mathrm{S})}$}

$\mathrm{O}$ cloreto de prata foi preparado tratando-se 5,920 g de $\mathrm{AgNO}_{3}$ em solução com 2,040 g de $\mathrm{NaCl}$ aquoso. O sólido branco de $\mathrm{AgCl}$ obtido foi lavado com água desionizada e seco em ambiente escuro, à temperatura ambiente por 2 dias.

Diversas proporções ponderais de resina poliéster e cloreto de prata foram misturadas em frascos de polietileno. Após homogeneização manual, $0,5 \mathrm{~mL}$ do catalisador (peróxido de metiletilcetona) foram adicionados. A mistura foi deixada à temperatura ambiente por 3-4 h. O sólido rígido obtido foi quebrado com martelo e um moinho multiuso Tecnal (mod. TE 631/1) foi utilizado para obter partículas de menor granulometria. A seleção do tamanho das partículas foi realizada usando-se peneiras com granulometria apropriada.

Cerca de $350 \mathrm{mg}$ das partículas de poliéster com granulometria entre 100-350 $\mu \mathrm{m}$ contendo $\mathrm{AgCl}$ foram introduzidas, por aspiração com uma seringa, dentro de tubos de polietileno (d.i. 2,0 mm e vários comprimentos) com uma das extremidades vedada com lã de vidro para prevenir a saída das partículas da coluna.

\section{Sistema de análise por injeção em fluxo}

O diagrama esquemático do sistema de análise por injeção em fluxo utilizado está representado na Figura 2. Alíquotas de $375 \mu \mathrm{L}$ (soluções de referência ou amostras) foram injetadas na solução transportadora $\left(\mathrm{NaOH} 0,01 \mathrm{~mol} \mathrm{~L}^{-1}\right)$ utilizando-se um injetor comutador-manual. Soluções de dipirona com concentrações variando entre 5,0 x $10^{-4}$ e 2,5 x $10^{-3} \mathrm{~mol} \mathrm{~L}^{-1}$, quando injetadas na solução transportadora, passam pelo reator em fase sólida reduzindo os íons $\mathrm{Ag}^{+}$a uma suspensão coloidal de prata metálica $\left(\mathrm{Ag}^{0}\right)$, que é liberada pela reação e monitorada turbidimetricamente em $425 \mathrm{~nm}$ (Esquema 1).

\section{RESULTADOS E DISCUSSÃO}

\section{Estudos preliminares}

Diversas soluções foram avaliadas como solução transportadora para verificar seu efeito sobre o sinal analítico. Nestes experimentos, a vazão da solução transportadora foi de $3,0 \mathrm{~mL} \mathrm{~min}{ }^{-1}$, 


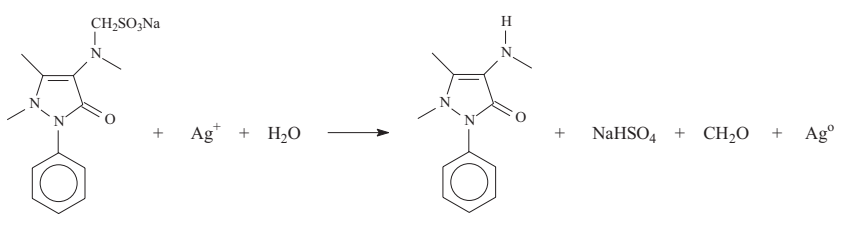

Esquema 1.

volume de amostragem de $250 \mu \mathrm{L}$, percurso analítico de $50 \mathrm{~cm}$ e um reator em fase sólida $(7,0 \mathrm{~cm}$ x 2,0 mm d.i.; tamanho de partícula 100-350 $\mu \mathrm{m}$ contendo $\mathrm{AgCl}$ imobilizado em resina poliéster na proporção 1:1). Soluções como borato de sódio $1,0 \times 10^{-4} \mathrm{~mol} \mathrm{~L}^{-1}$, tampão borato $0,10 \mathrm{~mol} \mathrm{~L}^{-1}(\mathrm{pH} 8,0$ e 9,0), tampão carbonato $0,10 \mathrm{~mol} \mathrm{~L}^{-1}(\mathrm{pH} 8,0 ; 9,0$ e 10$)$ e soluções de $\mathrm{NaOH}\left(1,0 \times 10^{-4} ; 1,0\right.$ x $10^{-3} ; 1,0 \times 10^{-2}$ e $1,0 \times 10^{-1} \mathrm{~mol} \mathrm{~L}^{-1}$ ) foram testadas e avaliadas quanto à repetibilidade, reprodutibilidade, estabilidade da linha base e tempo de limpeza do sistema FIA. Os melhores resultados em termos de sensibilidade e repetibilidade dos sinais de absorbância foram encontrados utilizando-se solução de $\mathrm{NaOH} 0,01 \mathrm{~mol} \mathrm{~L}^{-1}$ como solução transportadora. Em soluções tamponadas, os sinais de absorbância foram em torno de $70 \%$ menores apesar de se ter obtido maior estabilidade da linha base e menor tempo de limpeza. Em virtude dos melhores resultados em termos de magnitude do sinal analítico serem obtidos em meio básico, investigou-se a possibilidade da formação de $\mathrm{AgOH}$, ou mesmo $\mathrm{Ag}_{2} \mathrm{O}$, no reator e estes reagirem com a dipirona. Para avaliar este efeito, foram testados reatores compostos por $\mathrm{AgOH}$ e $\mathrm{Ag}_{2} \mathrm{O}$ no sistema em fluxo proposto, para uma solução de dipirona $1,0 \times 10^{-3} \mathrm{~mol} \mathrm{~L}^{-1}$. Tanto para $\mathrm{AgOH}$ como para $\mathrm{Ag}_{2} \mathrm{O}$, os sinais de absorbância decresceram cerca de $40 \%$ em comparação àqueles quando usado um reator com $\mathrm{AgCl}$ nas mesmas condições.

Em análises turbidimétricas, a adição de colóides protetores ou sufactantes é muitas vezes requerida com a finalidade de se obter uma eficiente nucleação uniforme, melhorando a reprodutibilidade das medidas ${ }^{27}$. O efeito dos surfactantes polietilenoglicol e etilenoglicol, além do EDTA, para uma concentração de dipirona de $1,0 \times 10^{-3} \mathrm{~mol} \mathrm{~L}^{-1}$ foi avaliado sobre o sinal analítico. $\mathrm{O}$ uso destas soluções não surtiu qualquer diferença nas medidas de absorbância, independente da concentração adotada. Em virtude desses resultados, optou-se por não utilizar nenhum outro reagente no sistema proposto.

\section{Estudo dos parâmetros do reator em fase sólida}

Os principais fatores que afetam o desempenho do reator em fase sólida como composição em massa entre o $\mathrm{AgCl}$ e a resina de poliéster, diâmetro interno, comprimento do reator e tamanho das partículas foram avaliados. Com o objetivo de minimizar os efeitos de compactação do reator ao longo do tempo, submeteu-se o mesmo a uma passagem contínua da solução transportadora por volta de 15 min antes das injeções. Em termos práticos, o sinal analítico estabilizou-se após as primeiras 20-30 injeções das soluções de dipirona.

Três diferentes composições em massa entre $\mathrm{AgCl}$ e a resina de poliéster, 12,$5 ; 25,0$ e $50,0 \%(\mathrm{~m} / \mathrm{m})$ foram investigadas para verificar o efeito do aumento da concentração de íons $\mathrm{Ag}^{+}$na determinação de dipirona. Observou-se um aumento da sensibilidade com o aumento da concentração de íons $\mathrm{Ag}^{+}$incorporada à resina. Como a composição ponderal de $50,0 \%(\mathrm{~m} / \mathrm{m})$ resultou na máxima carga de $\mathrm{AgCl}_{(\mathrm{s})}$ que pode ser agregada à matriz polimérica de poliéster, essa foi adotada em todo o trabalho.

O tamanho das partículas introduzidas no reator em fase sólida também afeta sua eficiência. Considerando os resultados obtidos nos trabalhos anteriores do grupo ${ }^{28-31}$, duas faixas de tamanhos de partículas foram investigadas neste trabalho (100-350 e 350-500 $\mu \mathrm{m})$. A faixa de 100-350 $\mu \mathrm{m}$ resultou em uma melhor sensibilidade, provavelmente devido à maior superfície de contato das partículas contendo o reagente imobilizado com a zona de amostra injetada. Além disso, esse tamanho de partícula não apresenta dificuldades operacionais para empacotamento dos reatores ou problemas de pressão hidrodinâmica.

$\mathrm{O}$ efeito do diâmetro interno da coluna $(1,0 ; 1,5 ; 2,0$ e $3,0 \mathrm{~mm})$ foi também avaliado em colunas preparadas com o mesmo comprimento $(7,0 \mathrm{~cm})$ e tamanho de partícula $(100-350 \mu \mathrm{m})$. Colunas com 2,0 mm de diâmetro interno resultaram em uma melhor sensibilidade e estabilidade da linha base. Colunas com menores diâmetros internos apresentaram uma maior pressão hidrodinâmica, dificultando assim o fluxo das soluções. Por outro lado, as colunas com 3,0 cm de diâmetro interno apresentaram baixa reprodutibilidade do sinal analítico devido, provavelmente, à maior dispersão da zona de amostra. Além disso, é mais fácil reproduzir a preparação de colunas com 2,0 mm de diâmetro interno. Sendo assim, o diâmetro interno de 2,0 $\mathrm{mm}$ foi escolhido.

O tempo de reação entre a zona de amostra e o $\mathrm{AgCl}$ imobilizado no reator em fase sólida depende de dois fatores: vazão da solução transportadora e comprimento do reator em fase sólida. A influência do comprimento do reator foi avaliada na faixa de 3,0 a $14 \mathrm{~cm}$ com uma vazão da solução transportadora de $3,0 \mathrm{~mL} \mathrm{~min}^{-1}$ (Figura 3). A magnitude de absorbância aumentou gradualmente, no entanto, reatores com comprimentos superiores a $8,5 \mathrm{~cm}$ não tiveram um aumento significativo do sinal, tendo ainda como agravante o aumento do desvio padrão entre as medidas e um tempo maior de limpeza devido, principalmente, a um aumento acentuado da pressão hidrodinâmica no sistema. Desta maneira, reatores de $8,5 \mathrm{~cm}$ e 2,0 $\mathrm{mm}$ (d.i.) empacotados com $\mathrm{AgCl}$ imobilizado em resina poliéster na proporção de $50,0 \%(\mathrm{~m} / \mathrm{m})$ com tamanho de partículas na faixa de 100-350 $\mu \mathrm{m}$ foram então utilizados no restante do trabalho.

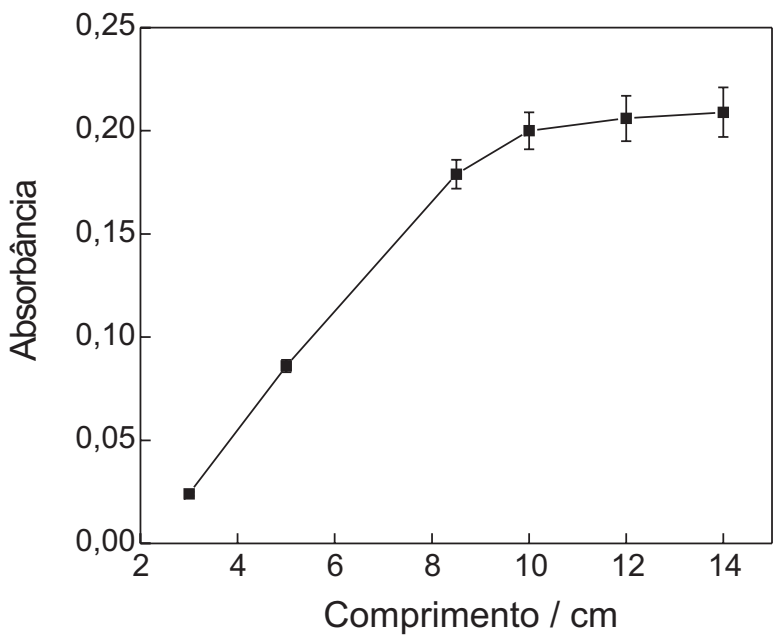

Figura 3. Efeito do comprimento do reator em fase sólida sobre o sinal analítico para uma concentração de dipirona 1,0 mmol $L^{-1}(n=5)$, solução transportadora $\mathrm{NaOH}$ 0,01 $\mathrm{mol} \mathrm{L}^{-1}$ a uma vazão de 2,0 $\mathrm{mL} \mathrm{min}^{-1}$, alça de amostragem de $375 \mathrm{~mL}$ e bobina helicoidal de $50 \mathrm{~cm}$

Após a seleção dos parâmetros ideais com relação ao reator em fase sólida, manteve-se o mesmo reator durante as demais etapas de desenvolvimento do sistema para avaliar seu tempo de vida útil. Durante um período de 6 dias, foram realizadas cerca de 500 injeções de dipirona no intervalo de concentração de $2,5 \times 10^{-4}$ a 2,0 x 
$10^{-3} \mathrm{~mol} \mathrm{~L} \mathrm{~L}^{-1}$ e obtidas curvas analíticas periódicas. Comparando-se as inclinações destas curvas, observou-se uma queda nos sinais de absorbância por volta de $30 \%$ da resposta inicial após cerca de 500 injeções. Isso ocorre principalmente devido à formação de $\mathrm{Ag}_{2} \mathrm{O}$ no reator que atua como um "agente passivador", diminuindo muito a eficiência da reação. Na prática, o melhor é a obtenção de uma curva analítica em média, por volta de 100-300 injeções.

\section{Estudo dos parâmetros do sistema em fluxo}

$\mathrm{O}$ volume da amostra foi avaliado variando-se o comprimento da alça de amostragem entre 12,5 e $100 \mathrm{~cm}(62,5$ e $500 \mu \mathrm{L})$ (Figura 4). A magnitude da absorbância aumentou gradativamente até $375 \mu \mathrm{L}$, permanecendo constante em volumes superiores. Isso se deve ao fato do volume da amostra ser superior ao volume do reator, não havendo assim uma interação efetiva entre sólido e solução. Desse modo, a quantidade de dipirona contida na alça de amostragem de $375 \mu \mathrm{L}$ é a quantidade máxima para que haja uma reação efetiva para as dimensões do reator escolhido. Sendo assim, selecionou-se um volume de $375 \mu \mathrm{L}$ de amostra para o restante do trabalho por apresentar, também, uma boa repetibilidade dos sinais.

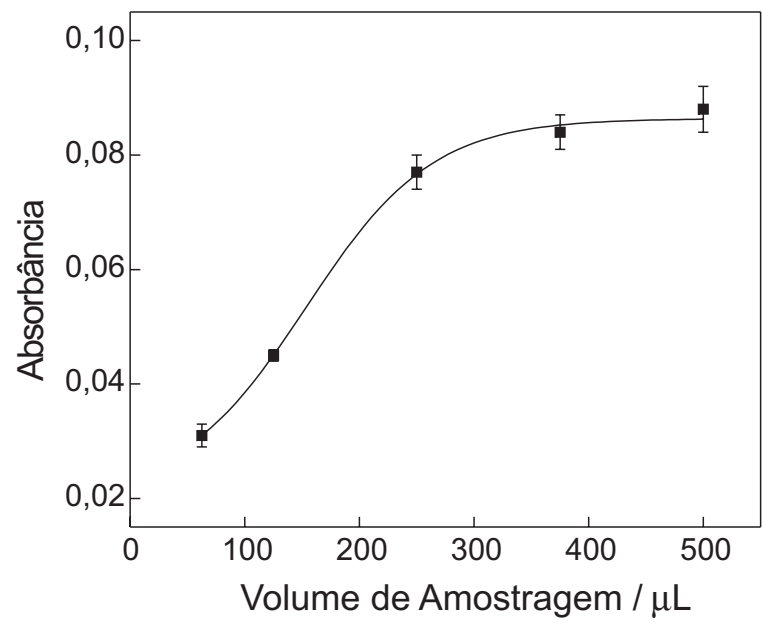

Figura 4. Efeito do volume da amostra sobre o sinal analítico para uma concentração de dipirona $1,0 \mathrm{mmol} \mathrm{L}^{-1}$, a $25^{\circ} \mathrm{C}(n=5)$

A Figura 5 mostra o efeito da vazão da solução transportadora variando de 1,0 a $5,1 \mathrm{~mL} \mathrm{~min}^{-1}$ sobre o sinal analítico. Os sinais de absorbância foram decrescendo significativamente como o aumento da vazão devido, principalmente, ao menor tempo de contato entre a zona de amostra e o reator contendo $\mathrm{AgCl}(\mathrm{s})$. A vazão de $2,0 \mathrm{~mL}$ $\min ^{-1}$ foi escolhida como ótima, por apresentar uma boa combinação entre sensibilidade e freqüência de amostragem e, também, estabilidade da linha base.

A influência da bobina helicoidal sobre o sinal analítico foi estudada na faixa de 50 a $200 \mathrm{~cm}$. Os valores de absorbância decresceram com o aumento do comprimento, provavelmente devido à dispersão do produto formado na reação com o reator em fase sólida. Sendo assim, optou-se por usar um tamanho mínimo (50 $\mathrm{cm})$ como bobina helicoidal neste trabalho.

\section{Estudo de interferentes em potencial e teste de recuperação}

O efeito de interferentes em potencial na determinação de dipirona em formulações farmacêuticas foi avaliado para excipientes normalmente presentes em amostras comerciais.

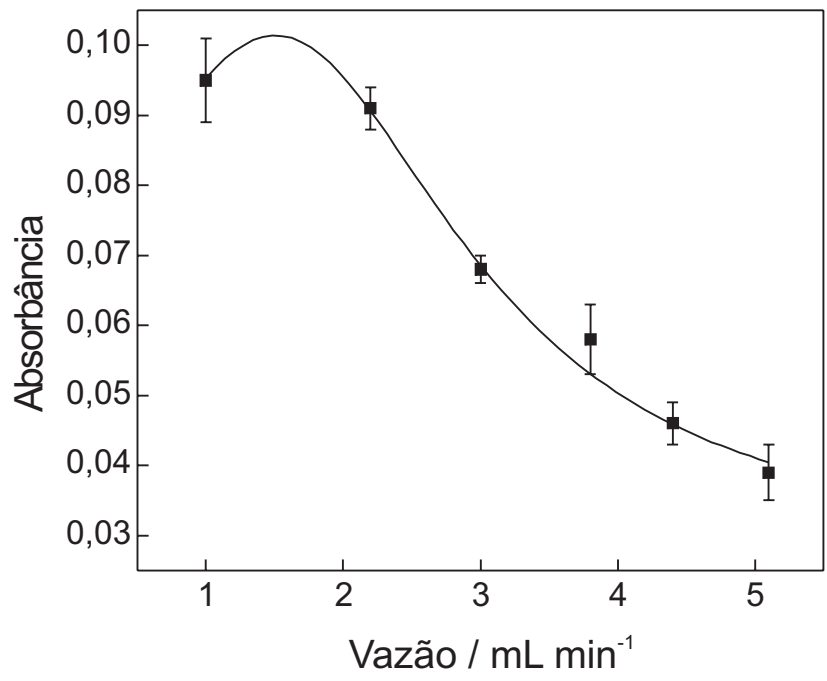

Figura 5. Efeito da vazão da solução transportadora sobre o sinal analítico para uma concentração de dipirona $1,0 \mathrm{mmol} \mathrm{L}^{-1}$, a $25^{\circ} \mathrm{C}(n=5)$.

Nesses experimentos foram utilizadas soluções de referência de $1,0 \times 10^{-3} \mathrm{~mol} \mathrm{~L}^{-1}$ de dipirona com cada um dos possíveis interferentes (sorbitol, cloreto de sódio, sacarina, glicerina, sacarose, lactose, EDTA e bissulfito de sódio) em concentrações de 1,0 x $10^{-3}$ e $1,0 \times 10^{-2} \mathrm{~mol} \mathrm{~L}^{-1}$. Nenhuma das substâncias investigadas causou qualquer interferência na determinação de dipirona com exceção do bissulfito de sódio, que causou uma interferência positiva da ordem de 3,0 e $13 \%$ quando presente em concentrações igual ou 10 vezes superior à de dipirona, respectivamente. Como nos produtos farmacêuticos o teor de bissulfito de sódio é bem menor que o teor de dipirona, amostras contendo este composto associado puderam ser analisadas sem maiores problemas.

A Tabela 1 apresenta os resultados dos testes de adição e recuperação do analito, em três concentrações diferentes de dipirona adicionadas a três amostras de formulações farmacêuticas e os resultados comparados com aqueles obtidos com as amostras não adicionadas do padrão. Os valores percentuais de recuperação variaram entre 96,0 e 102\% sugerindo, assim, ausência de interferência da matriz no procedimento proposto.

\section{Curva analítica e aplicações}

O método proposto foi aplicado para determinação de dipirona em produtos farmacêuticos. Soluções de referência de dipirona e soluções das amostras foram injetadas no sistema FIA apresentado na Figura 2. Os resultados obtidos estão apresentados na Tabela 2 .

A curva analítica foi linear no intervalo de concentração de dipirona entre 5,0 x 10-4 e 2,5 x 10-3 $\mathrm{mol} \mathrm{L}^{-1}(\mathrm{~A}=0,011+68,6 \mathrm{C}$; $\mathrm{r}=0,9990$, onde A é a absorbância e C, a concentração de dipirona em mol L ${ }^{-1}$ ) com um limite de detecção de $1,3 \times 10^{-4}$ mol L ${ }^{-1}\left(3 \sigma_{\mathrm{B}}\right.$ /inclinação). $\mathrm{O}$ desvio padrão relativo de 10 determinações sucessivas de dipirona $1,0 \times 10^{-3} \mathrm{~mol} \mathrm{~L}^{-1}$ foi de $1,8 \% \mathrm{e}$ a freqüência de amostragem alcançada foi de 45 determinações por hora.

Os resultados obtidos empregando-se o método proposto estão em boa concordância com aqueles obtidos pelo procedimento oficial da farmacopéia brasileira ${ }^{26}$ confirmando, assim, uma boa precisão do procedimento FIA desenvolvido. 
Tabela 1. Estudo de adição e recuperação de dipirona adicionada a formulações farmacêuticas empregando o sistema em fluxo com detecção turbidimétrica

\begin{tabular}{cccc}
\hline & \multicolumn{2}{c}{ Dipirona* $/ \times 10^{-3} \mathrm{~mol} \mathrm{~L}^{-1}$} & \\
\cline { 2 - 3 } Amostra & Adicionado & Recuperado & Recuperação/\% \\
\hline \multirow{2}{*}{ A } & 0,50 & $0,51 \pm 0,02$ & 102 \\
& 1,00 & $0,98 \pm 0,02$ & 98,0 \\
& 1,50 & $1,46 \pm 0,03$ & 97,3 \\
B & 0,50 & $0,49 \pm 0,01$ & 98,0 \\
& 1,00 & $0,97 \pm 0,02$ & 97,0 \\
C & 1,50 & $1,48 \pm 0,02$ & 98,7 \\
& 0,50 & $0,50 \pm 0,01$ & 100 \\
& 1,00 & $0,97 \pm 0,03$ & 97,0 \\
& 1,50 & $1,44 \pm 0,02$ & 96,0 \\
\hline
\end{tabular}

$* \mathrm{n}=5$

Tabela 2. Determinação de dipirona em produtos farmacêuticos empregando-se o sistema FIA turbidimétrico proposto e o método iodimétrico

\begin{tabular}{cccc}
\hline Amostras & Referência & Proposto & Er/\% \\
\hline A & $471 \pm 7$ & $478 \pm 3$ & $+1,5$ \\
B & $505 \pm 3$ & $478 \pm 3$ & $-5,3$ \\
C & $466 \pm 6$ & $459 \pm 2$ & $-1,5$ \\
D & $469 \pm 5$ & $482 \pm 2$ & $+2,8$ \\
\hline
\end{tabular}

valores em $\mathrm{mg} \mathrm{mL}^{-1} ; \mathrm{Er}=$ Proposto vs Referência

\section{CONCLUSÕES}

O sistema FIA de linha única utilizando um reator contendo $\mathrm{AgCl}$ imobilizado em resina poliéster mostrou ser uma metodologia simples, rápida e com boa precisão na análise de dipirona em produtos farmacêuticos, além de dispensar qualquer pré-tratamento das amostras, inclusive aquelas contendo corantes.

\section{AGRADECIMENTOS}

À FAPESP, CAPES e ao CNPq, pelo suporte financeiro e à Boheringer Ingelheim do Brasil pela dipirona sódica cedida, bem como ao CNPq pela bolsa de mestrado de V. G. Bonifácio e à FAPESP pelas bolsas de mestrado de L. H. Marcolino Jr. (Proc. no. 00/14988-8) e de pós-doutorado de M. F. S. Teixeira (Proc no. 00/ 02997-2)

\section{REFERÊNCIAS}

1. Korolkovas, A.; Burckhalter, J. H.; Química Farmacêutica, $1^{\text {a }}$ ed., Guanabara Koogan: Rio de Janeiro, 1988, p. 352.

2. Vassileva-Alexandrova, P.; Shishmanov, P. S.; Anal. Chem. 1975, 47, 1432.

3. Sakiara, K. A.; Pezza, L.; Melios, C. B.; Pezza, H. R.; De Moraes, M.; Il Farmaco 1999, 54, 629

4. Bautista, J. A. G.; Zamora, L. L.; Garcia-Mateo, J. V.; Martínez-Calatayud, J.; Anal. Lett. 1996, 29, 2667.

5. Qureshi, S. Z.; Saeed, A.; Haque, S.; Microchem. J. 1990, 41, 362.

6. Qureshi, S. Z.; Saeed, A.; Hassan, T.; Talanta 1989, 36, 869.

7. Pereira, A. V.; Penckowski, L.; Vosgerau, M.; Sassa, M. F.; Fatibello-Filho, O.; Quim. Nova 2002, 25, 553.

8. Buhl, F.; Hachula, U.; Chem. Anal. (Warsaw, Pol.) 1984, 29, 483

9. Buhl, F. Hachula, U. Chem. Anal. (Warsaw, Pol.) 1981, 26, 395.

10. Vassileva-Alexandrova, P.; Mikrochim. Acta 1976, 2, 247.

11. Abdine, H.; Soliman, S. A.; Morcos, M. G.; J. Pharm. Science 1973, 62, 1834.

12. Perez-Ruiz, T.; Martinez-Lozano, C.; Tomas, V.; Carpena, J.; Microchem. J. 1993, 47, 296.

13. Perez-Ruiz, T.; Martinez-Lozano, C.; Tomas, V.; J. Pharm. Biomed. Anal. 1994, 12, 1109.

14. Matos, R. C.; Angnes, L.; Araujo, M. C. U.; Saldanha, T. C. B.; Analyst 2000, 125, 2011.

15. Munoz, R. A. A.; Matos, R. C.; Angnes, L.; J. Pharm. Sci. 2001, 90, 1972.

16. Huang, Y. M.; Zhang, C.; Zhang, X. R.; Zhang, Z. J.; J. Pharm. Biomed. Anal. 1999, 21, 817.

17. Huang, Y. M.; Zhang, C.; Zhang, X. R.; Zhang, Z. J.; Fresenius J. Anal. Chem. 1999, 365, 381.

18. Huang, Y. M.; Zhang, C.; Zhang, X. R.; Zhang, Z. J.; Anal. Lett. 1999, 32, 933.

19. Agundez, J. A. G.; Benitez, J.; Ther. Drug Monit. 1996, 18, 104.

20. Tulaganov, A. A.; Mikhalev, A. V.; Osokina, L. M.; Arzamastsev, A. P.; Khimiko-Farm. Zh. 1992, 26, 76.

21. Agundez, J. A. G.; Martinez, C.; Martin, R.; Benitez, J.; Ther. Drug Monit. 1994, 16, 316.

22. Katz, E. Z.; Granit, L.; Drayer, D. E.; Levy, M.; J. Chromatogr. 1984, 305, 477.

23. Eddine, N. H.; Bressolle, F.; Mandrou, B.; Analyst 1982, 107, 67.

24. Martínez-Calatayud, J.; Flow Injection Analysis of Pharmaceuticals. Automation in the Laboratory, $1^{\text {st }}$ ed., Taylor \& Francis: Bristol, 1996.

25. Bergamin, F. H.; Reis, B. F.; Zagatto, E. A. G.; Anal. Chim. Acta 1978, 97, 427.

26. Farmacopéia Brasileira, $3^{\mathrm{a}}$ ed., Atheneu Ed.; São Paulo, 1977.

27. Heanes, D. L.; Anal. Lett. 1990, 23, 543.

28. Fatibello-Filho, O.; Marcolino-Junior, L. H.; Pereira, A. V.; Anal. Chim. Acta 1999, 384, 167.

29. Pereira, A. V.; Fatibello-Filho, O.; Talanta 1998, 47, 11.

30. Marcolino-Júnior, L. H.; Teixeira, M. F. S.; Pereira, A. V.; Fatibello-Filho, O.; J. Pharm. Biomed. Anal. 2001, 25, 393.

31. Teixeira, M. F. S.; Marcolino-Júnior, L. H.; Fatibello-Filho, O.; Il Farmaco 2002, 57, 215 